\title{
Prevalence of MRSA and its antibiotic susceptibility pattern in Ujjain, MP
}

\author{
L. Suresh Babu \\ Dept. of Microbiology, RD Gardi Medical College, Surasa, Ujjain, MP, India
}

Email address:

sureshbabu6831@yahoo.in

\section{To cite this article:}

L. Suresh Babu. Prevalence of MRSA and Its Antibiotic Susceptibility Pattern in Ujjain, MP. European Journal of Biophysics. Vol. 1, No. 5, 2013, pp. 37-40. doi: 10.11648/j.ejb.20130105.11

\begin{abstract}
Background and objectives: MRSA is known to cause community and hospital acquired infections due to its multiple drug resistance. The objectives of this study were to elucidate the prevalence of MRSA among clinical cases of both community and hospital acquired infections and to evaluate the invitro susceptibility pattern of different antibiotics against isolated MRSA strains. Methods: Clinical samples were collected from both inpatients and outpatients including swabs from operation theatres and ICUs and were subjected to MRSA screening by the conventional methods. Thus isolated MRSA were subjected to antibiotic susceptibility. Results \& interpretation: A total of 145 strains of Staphylococcus aureus were isolated. Among these 115 were from indoor patients, 21 from outdoor and 9 were of environmental sources. MRSA was $46 \%$ out of the total isolations and MSSA was 54\%. The maximum isolation of MRSA was from inpatients (49.5\%). The highest isolation rate of $92 \%$ of MRSA was reported from blood samples. Vancomycin was the most effective of all drugs used against MRSA strains in invitro susceptibility pattern. On the other hand moxifloxacin and gatifloxacin were the most effective against MSSA strains. Conclusions: MRSA is very much prevalent in this part of the country. A dip in the susceptibility of MRSA to vancomycin, prompts an immediate evolving of an alternate drug.
\end{abstract}

Keywords: MRSA, MSSA, SA, Methicillin, Environmental Sources

\section{Introduction}

Staphylococcus aureus is known to cause a variety of diseases, ranging from benign skin infections to life threatening illnesses like sepsis, endocarditis and osteomyelitis, throughout the world $(1,2)$. It has been reported as a major cause of community and hospital acquired infections (3). The inherent capacity to develop multiple drug resistance by the organism makes it a challenge to combat nosocomial infections $(4,5)$. Resistance to penicillinase resistant antibiotic like methicillin (MRSA) appeared first in 1961 (6). Since then MRSA has been reported from India and elsewhere $(7,8)$.

A constant surveillance for MRSA and vigorous treatment measures are required for controlling the endemics and epidemics of hospital acquired infections. This part of the country has not been investigated for MRSA before. So, this study has been undertaken to elucidate the prevalence of MRSA among various clinical cases and to evaluate their invitro susceptibility pattern against different antibiotics, in and around Ujjain, MP.

\section{Materials and Methods}

A total of 145 isolations of Staphylococcus aureus were made from various clinical samples, like, pus (which included swabs from blisters, beast abscesses and umbilical cord lesions), blood, throat swabs, urine, conjunctival swabs, aural swabs, nasal swabs, vaginal swabs and pleural aspirate, mostly, and also from swabs taken from operation theatres and neonatal intensive care units, from RD Gardi Medical College and Hospital in Ujjain, MP.

Of these total isolations, 115 were from clinical samples collected from patients admitted in the hospital for various ailments, 21 from specimens obtained outdoor patients and the remaining 9 from environmental sources as mentioned above. This work was carried out from August 2005 to July 2006.

The isolates were confirmed as Staphylococcus aureus by conventional methods, like, gram staining, mannitol fermentation and coagulase test (9). They were later subjected to antimicrobial susceptibility testing for methicillin ( $5 \mathrm{mcg} / \mathrm{disc})$ by modified Kirby-Bauer diffusion 
method on Mueller-Hinton agar by incubating for 24 hours at $35^{\circ} \mathrm{C}(10)$. All the methicillin resistant $S$. aureus (MRSA) strains were also tested with oxacillin $(1 \mathrm{mcg} / \mathrm{disc})$ for further confirmation.

Thus confirmed MRSA strains were later subjected to the various antibiotics based on NCCLS to study their susceptibility pattern (10). The antibiotics used were penicillin (10 units), ampicillin $(10 \mathrm{mcg})$, amoxicillin (20 $\mathrm{mcg}$ ), piperacillin (10 $\mathrm{mcg})$, cefprozil $(30 \mathrm{mcg})$, cefuroxime (30 mcg), gentamicin (10 mcg), amikacin (30 mcg), doxycycline $(30 \mathrm{mcg})$, tetracycline $(30 \mathrm{mcg})$, erythromycin (15 mcg), ofloxacin (5 mcg), vancomycin $(30 \mathrm{mcg})$, gatifloxacin $(5 \mathrm{mcg})$, ciprofloxacin $(5 \mathrm{mcg})$, moxifloxacin (5 mcg), chloramphenicol (30 mcg), cotrimoxazole $(1.25$ $\mathrm{mcg})$ and azithromycin (15 mcg), from HIMEDIA, Mumbai.

\section{Period and Place of Study}

Work has been carried out between August 2005 and July 2006 at the department of Microbiology, RD Gardi Medical College and Hospital, Ujjain, MP.

\section{Results}

Out of a total of 145 strains of Staphylococcus aueus strains isolated, $67(46 \%)$ were methicillin resistant (MRSA), and 78 (54\%) were methicillin sensitive (MSSA). Among the 67 MRSA, 57 were from indoor patients (hospital acquired), 6 (9\%) belonged to outdoor patients (community acquired) and the remaining $4(6 \%)$ were isolated from hospital environment sources (operation theatres and neonatal intensive care units). Of the 78 isolates of MSSA, $58(74 \%)$ were from indoor patients, 15 (19\%) from outdoor cases and $5(7 \%)$ from environmental sources (Table-I).

Clinical sample wise highest number of S.aureus strains isolated was from pus(81), followed by blood (24), urine (9), and throat swab and aural swab having 5 each, nasal and vaginal swab 4 each, conjunctival swab 3 and pleural aspirate 1. The total number of MRSA and MSSA strains isolated from the clinical samples is tabulated in Table-II.

Table 1. Distribution of MRSA and MSSA among inpatients, outpatients and environmental sources

\begin{tabular}{|c|c|c|c|}
\hline Sources & $\begin{array}{l}\text { No. S. aureus } \\
\text { isolated }\end{array}$ & MRSA & MSSA \\
\hline Inpatients & 115 & $57(49.5 \%)$ & $58(50.5 \%)$ \\
\hline Outpatients & 21 & $6(29 \%)$ & $15(71 \%)$ \\
\hline $\begin{array}{l}\text { Environmental } \\
\text { sources }\end{array}$ & 9 & $4(44 \%)$ & $5(56 \%)$ \\
\hline Total & 145 & $67(46 \%)$ & $78(54 \%)$ \\
\hline
\end{tabular}

Table 2. Distribution of MRSA and MSSA among various clinical samples

\begin{tabular}{llll}
\hline Samples & $\begin{array}{l}\text { No. of } \boldsymbol{S} \text {. aureus } \\
\text { isolated }\end{array}$ & MRSA & MSSA \\
\hline Pus & 81 & $26(32 \%)$ & $55(68 \%)$ \\
Blood & 24 & $22(92 \%)$ & $2(8 \%)$ \\
Throat swab & 5 & 4 & 1 \\
Urine & 9 & 7 & 2 \\
Conjunctival swab & 3 & 0 & 3 \\
Aural swab & 5 & 1 & 4 \\
Nasal swab & 4 & 1 & 3 \\
Vaginal swab & 4 & 3 & 1 \\
Pleural aspirate & 1 & 0 & 1 \\
\hline
\end{tabular}

On studying the antimicrobial susceptibility of MRSA, from indoor patients (57), it was found that the most number of MRSA strains, 34 (60\%), were susceptible to vancomycin followed by gatifloxacin 27 (47\%), azithromycin $26(46 \%)$ and moxifloxacin 23 (40\%). On the other hand the highest number of MRSA strains were resistant to penicillin $53(93 \%)$ followed by ampicillin 52 (91\%), amikacin 49 (86\%) and gentamicin 48 (84\%) (Figure 1).

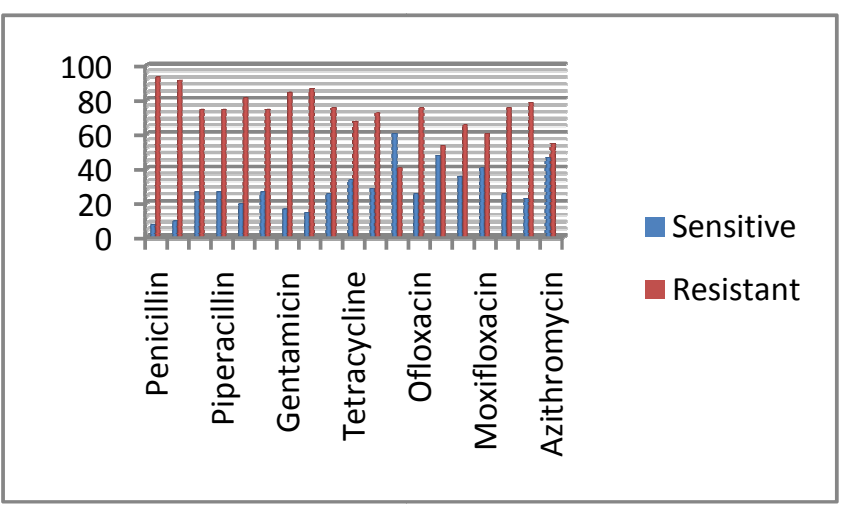

Figure 1. Sensitivity pattern of MRSA

Among the 6 MRSA isolated from outdoor patients, the most effective antibiotics were vancomycin, azithromycin, ciprofloxacin, cefuroxime and piperacillin. The most number of strains resistant were to penicillin, ampicillin, cotrimoxazole, doxycycline, erythromycin, tetracycline, cefpirome and chloramphenicol.

The antimicrobial susceptibility among a total of 58 isolations of MSSA from indoor patients, the most effective drugs were, gatifloxacin and moxifloxacin, 52 (90\%) of each, followed by vancomycin $45(78 \%)$ and cefprozil 44 $(76 \%)$. The most number of MSSA resistant were to penicillin $58(100 \%)$, followed by ampicillin 57 (98\%) and erythromycin 56 (97\%) (Figure 2). 


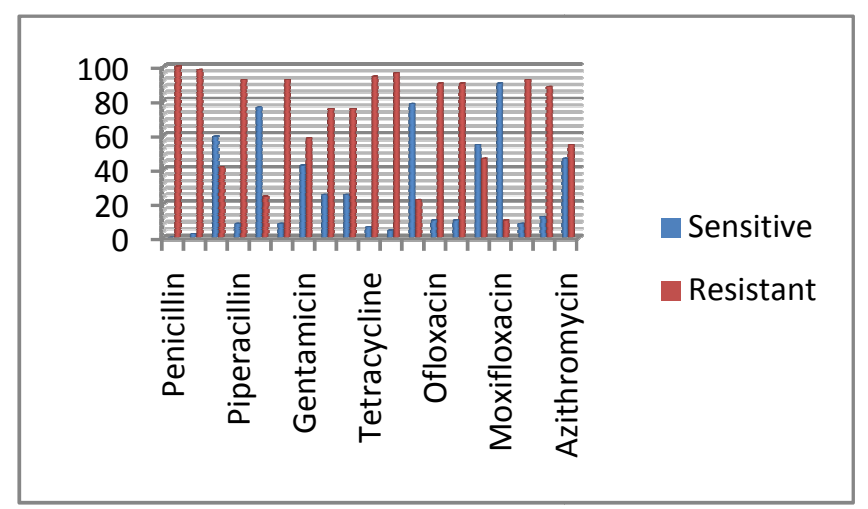

Figure 2. Sensitivity pattern of MSSA

Among the 15 MSSA outdoor isolations, the most effective antibiotic was cefprozil, followed by moxifloxacin and ciprofloxacin. The most number of strains were resistant to penicillin, followed by tetracycline and cefuroxime.

\section{Discussion}

There were no earlier reports of MRSA from this part of the country. Our study indicates a restively a higher prevalence $(46 \%)$ of MSRA among the total number of $S$. aureus strains isolated from various clinical cases. This observation is in correlation with the earlier studies in Assam and Karnataka $(11,12)$. Both outdoor and indoor patients were included in our study so as to understand the prevalence of MRSA among community acquired and nosocomial infections, respectively. The prevalence rate of MRSA among indoor isolations of $S$. aureus (49.5\%), is more than as reported by SK Mathur et al, 1994, though the outdoor rate of $29 \%$ is in correlation with their study (13). The presence of $5 \mathrm{MRSA}$ strains out of $9 \mathrm{~S}$. aureus isolated from environmental sources (operation theatres and ICUs), further strengthens the view of MRSA spread of nosocomial infections. The highest isolation of MRSA from blood $(92 \%)$ among all other clinical specimens is in the same view of Dalip K Kakru et al, 2003 (14). This may be attributed to the iatrogenic infection occurring among inpatients. Another interesting observation is the higher prevalence of MRSA among UTI, URTI and gynaecological cases as evidenced by their isolation from urine, throat swab and vaginal swab, respectively (Table II).

While studying the antibiotic susceptibility pattern among MRSA strains, we have included 19 antibacterials representing penicillins, cephalosporins, aminoglycosides, tetracyclines, macrolides, glycopeptides and quinolones, apart from chloramphenicol, cotrimoxazole and azithromycin as per NCCLS.

Though vancomycin is found to be the most effective drug $(60 \%)$ as like the earlier investigations (K Rajaduraipandi et al, 2006) (15), the number of MRSA strains responding to it has come down, which is a concern to evolve newer antibiotics to control the spread of infections due to MRSA.
While attempting to study the relative response of MSSA strains in comparison with MRSA, we found an increased susceptibility pattern. But, gatifloxacin and moxifloxacin have overtaken vancomycin as the most effective drugs, which is a variation from the earlier reports.

In conclusion, MRSA is very much rampant in this part of the country. Also the fact that decrease in the susceptibility of MRSA strains to vancomycin is a major concern to evolve a newer drug showing uniform sensitivity against these strains.

\section{References}

[1] Couto I, Melo-cristino J, Fernandes T, Garica N, Serrano MJ, Salgado A et al. Unusually large number of methicillin resistant Staphylococcus aureus clones in a Portuguese hospital. J Clin Microbiol 1995; 33: 2032-2035.

[2] Cox RA, Conquest C, Mallaghan C and Marples RR. A major outbreak of methicillin resistant $S$. aureus caused by new phage type (EMRSA-16). J Hosp Infect 1995; 29: 87-106.

[3] Vidhani S et al. Study of methicillin resistant S. aureus isolates from high risk patients. Indian $\mathrm{J}$ Med Microbiol 2001;19(2):87-90.

[4] Bajaj JK, Karyakarte RP, Kulkarni JD, Deshmukh A.B. Prevalence of resistant Staphylococcus aureus at Aurangabad. J Commun Dis1999; 31(3): 173-176.

[5] Sheagren JN. Staphylococcus aureus, the persistent pathogen. New Eng J Med1984; 310: 1368-1373.

[6] Jevons M Patricia. "Celbenin" - resistant Staphylococci. Br Med J. 1961 14;1(5219):124-125.

[7] Chaudary U, Anupama. Prevalence of methicillin resistant Staphylococcus aureus. Indian J Med Microbiol 1999;17(3):154-155.

[8] Michel M, Gutmann L. Methicillin resistant Staphylococcus and vancomycin resistant enterococci Enterococci, therapeutic realities and possibilities. Lancet 1997; 349: 1901-1906.

[9] Colle JG, Fraser AG, Marmion BP, Simmon A. Mackie and Mc Cartney's Practical Medical Microbiology. 14 th edition.Churchill Livingstone 1996; 254-256, 796-800.

[10] National Committee for Clinical Laboratory Standards (NCCLS). Performance standards for antimicrobial disk susceptibility tests. Approved standard M2-A7. Wayne (PA): NCCLS 2000.

[11] Majumder D, Sarma Bordoloi JN, Phukan AC, Mahanta J. Antimicrobial susceptibility pattern among methicillin resistant Staphylococcus isolates in Assam. Indian J Med Microbiol 2001;19(3):138-140.

[12] Hanumanthappa AR, Chandrappa NR, Rajasekharappa MG. Prevalence of methicillin resistant Staphylococcus aureus in Karnataka. Indian J Pathol Microbiol 2003;46(1):129-132.

[13] Mathur SK, Singhal S, Prasad KN, Kishore J, Ayyagari A. Prevalence of methicillin resistant Staphylococcus aureus (MRSA) in tertiary care hospital. Indian J Med Microbiol 1994;12(2):96-101. 
[14] Kakru DK, Sheikh A, Thaker MA, Wani T. Methicillin resistant Staphylococcus aureus: need for constant surveillance, stringent control and vigorous treatment measues. Indian J Pathol Microbiol 2003;46(1):121-123.
[15] Rajaduraipandi K, Mani KR, Panneerselvam K, Mani M, Bhaskar M, Manikandan P. Prevalence and antimicrobial susceptibility pattern of methicillin resistant Staphylococcus aureus: A multicentre study. Indian J Med Microbiol 2006;24(1):34-38. 